\title{
Hierarchical Models of Self-Concept across Genders and Sciences/Humanities for College Students in Taiwan
}

\author{
Mei-Shiu Chiu \\ Department of Education, National Chengchi University, 64 Zhinan Road Section 2, Taipei 11605, Taiwan
}

Correspondence should be addressed to Mei-Shiu Chiu; chium@nccu.edu.tw

Received 28 February 2013; Accepted 13 May 2013

Academic Editors: S. Cessna and C. C. Schifter

Copyright (C) 2013 Mei-Shiu Chiu. This is an open access article distributed under the Creative Commons Attribution License, which permits unrestricted use, distribution, and reproduction in any medium, provided the original work is properly cited.

\begin{abstract}
The aim of this study is to examine the hierarchical model of self-concept (SC) using two operations (Models A and B). In Model A, global self-concept (GSC) is the higher-order factor of field-specific self-concepts (FSCs). In Model B, GSC is the predictor of the correlated FSCs. The data of 28,824 college students are obtained from the national database for higher education in Taiwan. The two models are examined by the use of total-group hierarchical confirmatory factor analysis (HCFA) and structural equation modeling (SEM), respectively. Multigroup HCFA and SEM are used to test whether the two models demonstrate measurement equivalence and structural invariance across female and male college students and across college students coming from different fields of study. The results of the analysis reveal three major findings. (1) The data fit Models A-B well for all the students as a whole. (2) Females and males are found not to be equivalent in the structure of both models. (3) Females from female-dominated humanities fields (education and business) are different from those in male-dominated sciences fields (engineering and natural sciences), while males from the four fields are similar in both models.
\end{abstract}

\section{Introduction}

Self-concept is one of the major constructs in education, and appropriate development of self-concept has become one of the most major goals in education $[1,2]$. Theories on self-concept, however, are normally posited by Western scholars, and the operation of self-concept is dominated by instruments developed in Western culture (e.g., $[3,4])$, even though the theories and operations were supported by studies conducted for individuals form diverse cultures (e.g., [5-10]). Relatively few studies develop instruments suitable for the researchers' own cultures to address the issue of hierarchical and multidimensional structure of self-concept [11]. The aim of the study is to examine one of the major theories on self-concept using a different instrument and different subconstructs based on data from the official and continued large-scale database for higher education in Taiwan. The reoperation of a well-known theory on self-concept in Western culture is likely to advance the understanding of the theory and the culture in a non-Western culture.

Shavelson et al. [12] posited a theory of a hierarchical structure of self-concept (SC), in which self-concept is regarded as an organized, multifaceted, hierarchical, stable, developmental, evaluative, and differential network. The hierarchy can be depicted as a system with one single, highestorder general self-concept (GSC) and several lower-order field-specific self-concepts (FSCs), for example, academic, social, emotional, and physical self-concepts (SCs). A lowerorder FSC, such as academic SC, can be further divided into much lower-order FSCs, for example, verbal, math, and science SCs. Shavelson et al.s theory is supported by research results that show model fit using hierarchical confirmatory factor analysis, and those that reveal that domainspecific self-concepts have a more reliable relationship with important external criteria, such as academic achievement, than general self-concept [13-17]. Based on Shavelson et al.s theory, a revised Marsh/Shavelson model was posited by Marsh and Shavelson [18], and the results of empirical studies revealed that GSC consisted of three lower-order FSCs: math, English, and general school for school-level students [19-21]. As a result, later research on hierarchical models of SC generally focuses on the two core domains of math and verbal skills, which are concise constructs for 
SC but may not be suitable for tertiary education, in which multiple abilities are emphasized, and domains of knowledge are more differentiated than those in primary and secondary education.

Shavelson et al.s [12] emphasis on a "hierarchical structure" of SC allows us to draw an analogy between the structure of SC and that of a nation. The notion of an "organized, multifaceted, hierarchical, and differential" structure of SC tackles the issue of the roles that GSC (the central government) may play in its FSCs (the local governments). The GSC can play its role in FSCs in two ways or models: (1) GSC is virtually defined by, adapted to, and derived from its FSCs, and (2) GSC substantially leads, organizes, and controls its associated FSCs. On the other hand, the notion of a "stable, developmental, and evaluative" structure tackles the issue of the evolution of the hierarchical structure of SC as a response to individual, situational, and cultural factors. As inspired by research on academic SC (e.g., [22]), gender and fields of study (or domains of knowledge) may be two effective factors moderating the hierarchical relationships between GSC and FSCs. The purpose of this study, therefore, is to examine the two hierarchical models of SC and to explore the role of gender and fields of study in the two models.

\section{Two Hierarchical Models of Self-Concept}

2.1. The Hierarchical Model of SC Where GSC Is a HigherOrder Factor of FSCs. Marsh and his colleagues [16-18] base their research on Shavelson et al.s [12] seminal work and aim to examine the hierarchical structure of SC. The typical procedure they use for the examination of the hierarchical structure of SC is hierarchical confirmatory factor analysis (HCFA). Their findings generally support a hierarchical structure of SC, but the higher-order GSC still fails to be consistently or accurately defined by its lower-order or much lower-order FSCs [13].

Studies on gender differences in the hierarchical structure of SC generally support the invariance of factor loadings across genders $[14,23]$. The fact that females take responsibility for housework or child care and sacrifice personal interests or careers more than males [24], however, suggests that females may distinguish different motivations or FSCs better than males. In other words, males may view different FSCs as more similar than females.

There are also likely interactions between gender and fields of study because females are more sensitive to social messages, expectations, or pressures than males [25]. Taiwan has a confusion tradition, which advocates males' role in earning money and females' role for caring about people $[26,27]$. Natural sciences are viewed as less human and with higher salary than social sciences [28]. As such, males from different fields of study may be similar in the hierarchical structure of SC, but females from different fields of study may be different.

2.2. The Hierarchical Model of SC Where GSC Is a Predictor of Correlated FSCs. As stated previously, research findings fail to consistently support Shavelson et al.s [12] theory of a hierarchical structure of SC by the statistical procedure of HCFA. The inconsistent findings may raise a theoretical concern of complex-defined higher-order factors due to multiple levels of abstraction [29]. Another procedure to examine the hierarchy is likely to be the use of a path model, for example, structural equation modeling (SEM). Shavelson et al. has indicated that "changes at the lower levels of the hierarchy are probably attenuated by conceptualizations at higher levels (p. 414)," which suggests a leading effect of GSC on FSCs. In addition, the low correlations between FSCs (i.e., math and verbal SCs) for adolescents have triggered another line of research that focuses on the use of a path model for the relations between FSCs and other contextual variables (e.g., [30]). The use of a path model, with a GSC to be directly inferred from a specific scale and to have a substantial effect on FSCs, is likely to broaden the understanding of the hierarchical structure of SC posited by Shavelson et al.

The major difference between a hierarchical factor model and a path model lies in their conceptual operations of the hierarchical structure. A hierarchical factor model tests a measurement theory, and a path model tests a structural theory. In other words, we obtain a virtual GSC defined by its FSCs with a hierarchical factor model, and we use a GSC defined by actual items to test its ability to lead its FSCs with a path model. We can regard a hierarchical factor model as a tight way to test Shavelson et al.s [12] theory because the factor loadings of lower-order FSCs on the higher-order GSC all have to be significant. A path model is a relatively loose way to examine Shavelson et al.s theory, as the path coefficients leading from GSC to FSCs can vary so as to demonstrate differential regression effects of GSC on each of the FSCs. A path model, however, is a more flexible and convenient way than multigroup and multilevel analyses in (1) exploring the diverse relations between every SC in the hierarchy and other constructs and (2) discovering differential patterns of the hierarchy in response to different contexts or individuals, for example, fields of study and gender.

Although there may be low associations between some FSCs, for example, math and verbal SCs [13, 23, 30], fields or domain comparison does exist for related fields [31], since trans- or cross-domain learning is highly emphasized in higher education. As such, the associations between FSCs for college students are assumed.

Gender differences in the hierarchical structure of SC where GSC acts as the predictor of correlated FSCs may be inferred by the differential socialization hypothesis. GSC can be viewed as a long-term database of past experiences and social messages or expectations, for example, gender roles. Therefore, males with a high GSC may imply their SC in traditional male skills, such as repairing a radio, and females with a high GSC may imply their SC in traditional female skills like social skills. The differential socialization hypothesis, however, is not supported for the hierarchical structure of SC where GSC is the higher-order factor of FSCs by the statistical procedure of HCFA in Marsh's [14] study.

There are also likely interaction between gender and fields of study because females are more sensitive to social expectations. Similar rationales are stated in "the hierarchical model of SC where GSC is a higher-order factor of FSCs." 
TABLE 1: Summary of Hypotheses, Rationales, and Statistical Methods.

\begin{tabular}{|c|c|c|c|}
\hline Models & Model fit & Gender differences & Gender $\times$ field of study \\
\hline $\begin{array}{l}\text { Model A: } \\
\text { GSC as the factor of FSCs }\end{array}$ & H1: Model A fits data. & $\mathrm{H} 2$ : females $\neq$ males & $\begin{array}{l}\text { H3: females differ, and males are similar } \\
\text { across different fields. }\end{array}$ \\
\hline $\begin{array}{l}\text { Model B: } \\
\text { GSC as the predictor of } \\
\text { correlated FSCs }\end{array}$ & H4: Model B fits data. & H5: females $\neq$ males & $\begin{array}{l}\text { H6: females differ, and males are similar } \\
\text { across different fields. }\end{array}$ \\
\hline Rationales & $\begin{array}{l}\text { H1: GSC is the higher-order } \\
\text { factor of FSCs, which imply } \\
\text { that FSCs are correlated. } \\
\text { H4: GSC predicts } \\
\text { correlated FSCs. }\end{array}$ & $\begin{array}{l}\text { H2: females view FSCs as } \\
\text { different SCs more than males. } \\
\text { H5: GSC is reflective of } \\
\text { long-term social expectation of } \\
\text { gender roles. }\end{array}$ & $\begin{array}{l}\text { H3: Females are more sensitive to } \\
\text { (short-term) social factors than males. } \\
\text { H6: females are more sensitive to } \\
\text { (short-term) social factors than males. }\end{array}$ \\
\hline Statistical methods & $\begin{array}{l}\text { H1: total-group HCFA } \\
\text { H4: total-group SEM }\end{array}$ & $\begin{array}{l}\text { H2: multigroup HCFA } \\
\text { H5: multigroup SEM } \\
\text { (for both genders from all fields) }\end{array}$ & $\begin{array}{l}\text { H3: multigroup HCFA } \\
\text { H6: multigroup SEM } \\
\text { (for both genders from different fields) }\end{array}$ \\
\hline
\end{tabular}

H1 H6: Hypothesis 1 Hypothesis 6; GSC: general self-concept; FSCs: field-specific self-concepts; SCs: self-concepts; HCFA: hierarchical confirmatory factor analysis; SEM: structural equation modeling. Other information about Models A-B can be found in Figure 1.

2.3. Summary of Hypotheses. Two hierarchical models of SC are posited to interpret the hierarchical structure of SC proposed by Shavelson et al. [12]. Each of the models operates through different psychological mechanisms (Table 1).

In Model A (Figure 1) of this study, a GSC is a higherorder factor defined by six FSCs (six lower-order factors), each of which is defined by two items. The FSCs are potentially correlated because of the common higher-order factor, that is, GSC; as such, Model A will fit data (Hypothesis 1). There are gender differences in Model A (Hypothesis 2) because Model A is likely to reflect that females view FSCs as different SCs more than males. There is also likely to be interactions between genders and fields of study in Model A, with females being different and males being similar from different fields of study (Hypothesis 3) because females are more sensitive to (short-term) social factors than males. Total-group and multigroup HCFA are used to examine Hypotheses 1-3 for all students and both genders from different fields of study.

Model B has an actual GSC (defined by three items) predicting six FSCs (each defined by two items) that are correlated to each other, so Model B should fit data (Hypothesis 4). There are gender differences in Model B (Hypothesis 5) because Model B highlights the effect of GSC as a database of long-term experiences of gender roles on FSCs. There are also interactions between genders and fields of study in Model B, with females being different and males being similar from different fields of study (Hypothesis 6) because females are more sensitive to (short-term) social factors than males. Hypotheses 3 and 6 are based on the same rationales.

\section{Method}

3.1. Data Source and Participants. The data for this study come from the Integrated Higher Education Database System (IHEDS) in Taiwan, complied by National Taiwan Normal University [32]. First- and third-year college students in Taiwan are selected to provide information through a questionnaire on their sociobiographic background, study experiences, and personal viewpoints since 2003. The IHEDS employs a stratified sampling design based on university types and fields of study, and at least 100 students are selected from each university in Taiwan. The participants of the IHEDS have been gradually extended to include fourth-year college students, graduates, faculty, and administrative staff in higher education.

The data from third-year college students $(N=28,824$, $55 \%$ females) attending 140 universities in 2003 were used for the present investigation because these students had received higher education for a relatively short-term period of three years, compared with their past experiences. A special focus is placed on students from four fields of study: engineering ( $N=5,929,14 \%$ females), natural sciences $(N=901,35 \%$ females), education ( $N=1,026,71 \%$ females), and business ( $N=6,629,52 \%$ females) (total $N=14,485$, $46 \%$ females, Table 3 ). The numbers of students indicate that more males studied engineering and natural sciences, while more females studied education and business in Taiwan, a phenomenon widely documented in the literature of higher education [33].

3.2. Measures. All the items of the measures were written in Mandarin, the official language of Taiwan, when the survey was administered. The items were translated into English for the present paper.

3.2.1. General Self-Concept (GSC). The survey asked students to rate their SC in general on a four-point Likert scale ( $1=$ strongly disagree; $4=$ strongly agree). The measure of GSC includes the following three items: I think I am a valuable person, at least as valuable as others. I feel that I have many advantages. Generally speaking, I am satisfied with myself.

3.2.2. Field-Specific Self-Concepts (FSCs). Students were asked to indicate their ability in six specific fields, classified according to Holland's theory [34], on a two-point scale ( 1 = bad at; 2 = good at). Holland's theory maintains that there are six types of vocations/interests (realistic, investigative, 


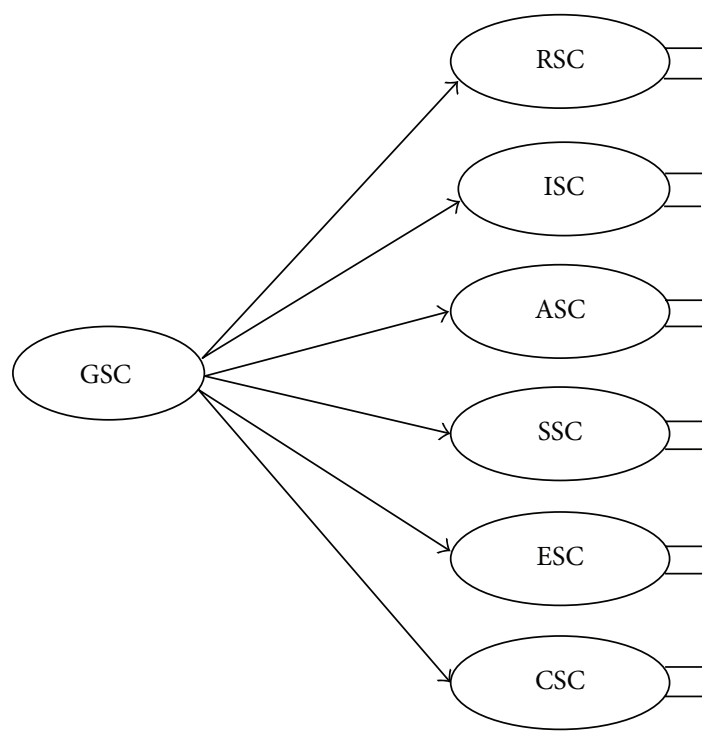

(a) Model A. GSC as the higher-order factor of FSCs

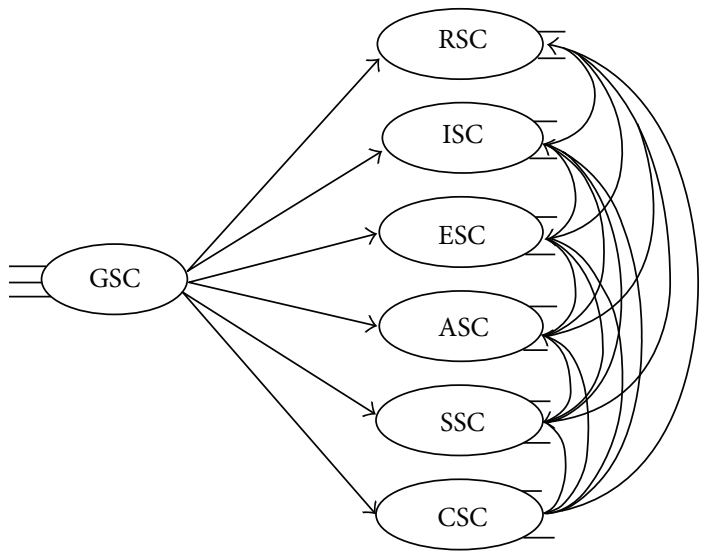

(b) Model B. GSC as the predictor of correlated FSCs

FIGURE 1: Two hierarchical models of self-concept. GSC: general self-concept; FSCs: field-specific self-concepts, including RSC, ISC, ASC, SSC, ESC, and CSC here; RSC: realistic self-concept; ISC: investigative self-concept; ASC: artistic self-concept; SSC: social selfconcept; ESC: enterprising self-concept; CSC: conventional selfconcept; “ $\rightarrow$ ": predictive path; “ $\leftrightarrow$ ”: correlation.

artistic, social, enterprising, and conventional) and parallel environments, for example, departments in the university and vocations in the society [35]. Applying the constructs of Holland's interest types into those of ability-related concepts appears to be a rare practice.

The FSC included 12 items, with two items on each of the six FSCs as follows.

(1) Use tools such as machines, electric appliances, and carpentry devices (realistic self-concept (RSC)).

(2) Repair electric appliances, furniture, or pipes at home (RSC).

(3) Study literature on math, biology, physics, and chemistry (investigative self-concept (ISC)).

(4) Use math to solve practical problems (ISC).
(5) Write, paint, perform, or play musical instruments (artistic self-concept (ASC)).

(6) Do creative work (ASC).

(7) Interact with different types of people (social selfconcept (SSC)).

(8) Relieve people in distress (SSC).

(9) Lead and supervise others (enterprising self-concept (ESC)).

(10) Persuade others to work according to my methods (ESC).

(11) Organize messy data and files (conventional selfconcept (CSC)).

(12) Record details and sort records (CSC).

3.2.3. Demographic Data. The IHEDS provides participants' demographic data, such as gender and fields of study.

Student scores are converted into standardized $z$-scores with a mean of 0 and a standard deviation of 1 . The alpha reliability of the measures is .780 for GSC, .701 for RSC, .611 for ISC, .541 for ASC, .548 for SSC, .672 for ESC, and .781 for CSC. Confirmation factor analysis (CFA) was used to test a one-order factor model of the GSC and the six FSCs together. The results of CFA show that the factor loadings, uniqueness, and factor correlations are all significant (Table 2). Factor correlations vary from .054 (between RSC and CSC) to .747 (between SSC and ESC). The values of the fit indexes suggest that the one-order factor model fits data well $\left(\chi^{2}=1044.232\right.$, $P<.05 ; \mathrm{CFI}=.993$; NNFI $=.989$; RMSEA $=.022)$. The meaning of the fit indexes will be explained in the following statistical analysis.

3.3. Statistical Analysis. The analyses of CFA and SEM are performed using the software of LISREL 8.80 [36]. Detailed data analysis procedures and basic explanations of the analysis results can be found in M. Du Toit and S. H. C. Du Toit [37] and Jöreskog and Sörbom [38].

Model A in Figure 1 (Hypotheses 1-3) is examined using HCFA, which fits responses to six FSCs (lower-order factors) and then fits the FSCs to GSC (the higher-order factor). HCFA is a suitable statistical procedure for examining the hierarchical structure of SC, as suggested by Marsh et al. [16]. Models B (Hypotheses 4-6) are examined using SEM, which tests influences or effects of GSC on FSCs. Total-group HCFA or SEM is used to examine a model based on data from a group of people as a whole. Multigroup HCFA or SEM is suitable for testing moderating effects; in other words, it is employed to test whether the posited models demonstrate measurement equivalence and structural invariance across female and male college students and across college students coming from different fields of study.

The most commonly used goodness-of-fit index for CFA, HCFA, or SEM is $\chi^{2}$, and a nonsignificant $\chi^{2}$ represents a good fit. The $\chi^{2}$, however, will become very large because of large sample sizes, and therefore it is not a suitable fit index here $[39,40]$. A model fit can also be indicated by values of 
comparative fit index (CFI) or nonnormed fit index (NNFI) larger than .900 and values of root mean square error of approximation (RMSEA) smaller than $100[29,41]$.

\section{Results}

4.1. GSC as the Higher-Order Factor of FSCs. The model in which GSC acts as the higher-order factor of FSCs (Model A in Figure 1) fits data well, which supports Hypothesis 1, as indicated by the fit indexes obtained by HCFA for all students $(\mathrm{CFI}=.958$; NNFI $=.942>.900$; $\mathrm{RMSEA}=.051$ $<.100$ (Table 3)). In addition, all the factor loadings of GSC defined by six FSCs are positive and significant, a result consistent with Shavelson et al.s [12] theory and related research findings, for example, Marsh et al. [16].

Females and males are found not to be equivalent in the structure of Model A, a result supporting Hypothesis 2. In other words, the parameters obtained by multigroup HCFA appear not to fit the data across genders as indicated by the fit indexes, with CFI $=.730<.900$, NNFI $=.742<.900$, and RMSEA $=.102>.100$. Therefore, it is sensible to let both females and males tell their own stories. Total-group HCFA is performed for females and males, respectively, and the results reveal that Model A fits both the data of females (CFI $=.965>$ .900 , NNFI $=.952>.900$, and RMSEA $=.044<.100)$ and those of males $(\mathrm{CFI}=.970>.900, \mathrm{NNFI}=.959>.900$, and RMSEA $=.046<.100$ ) well. The results imply that the model in which GSC acts as the higher-order factor of FSCs is a suitable structure for both females and males, but the patterns of the structure are different between females and males. We can find that the factor loadings of GSC on the six FSCs for females $(.178, .227, .483, .854, .837$, and .415$)$ are weaker than those for males $(.324, .338, .594, .856, .878$, and .501$)$. The results suggest that females differentiate FSCs better than males.

Hypothesis 3 predicts that females from different fields of study are different and males from different fields of study are similar in Model A. A special focus is placed on the comparison between the two male-dominated fields (more males studying engineering and natural sciences than females) and the two female-dominated fields (more females studying education and business than males). The results of multigroup HCFA reveal that Model A does not fit females across the four fields $(\mathrm{CFI}=.783<.900$, NNFI $=.820<.900$, and RMSEA $=.107>.100)$, but Model A fits males across the same four fields $(\mathrm{CFI}=.915>.900$, NNFI $=.929>.900$, and RMSEA $=.061<.100$ ), a result supporting Hypothesis 3. A closer look at the results obtained by multigroup HCFA for each of the four fields reveals that females and males are similar if they are from the fields of engineering $(\mathrm{CFI}=.934$, $\mathrm{NNFI}=.936$, and RMSEA $=.057)$ and natural sciences $(\mathrm{CFI}=$ .908 , NNFI $=.912$, and RMSEA $=.056)$. Females and males, however, are different if they are from the fields of education $(\mathrm{CFI}=.696, \mathrm{NNFI}=.709$, and RMSEA $=.123)$ and business $(\mathrm{CFI}=.745, \mathrm{NNFI}=.756$, and RMSEA $=.110)$. If we combine this with the previous result that females from the four fields are different and males are similar, we can infer that females are more sensitive to or reflective of their study fields than males.
TABle 2: Parameter estimates obtained by confirmatory factor analysis.

\begin{tabular}{|c|c|c|c|c|c|c|c|c|}
\hline \multirow{2}{*}{ Measures } & \multicolumn{7}{|c|}{ Factor loadings } & \multirow{2}{*}{ Uniqueness } \\
\hline & GSC & RSC & ISC & ASC & SSC & ESC & CSC & \\
\hline \multicolumn{9}{|l|}{ GSC } \\
\hline Item 1 & .765 & & & & & & & .415 \\
\hline Item 2 & .840 & & & & & & & .294 \\
\hline Item 3 & .612 & & & & & & & .626 \\
\hline \multicolumn{9}{|l|}{ RSC } \\
\hline Item 1 & & .775 & & & & & & .400 \\
\hline Item 2 & & .696 & & & & & & .516 \\
\hline \multicolumn{9}{|l|}{ ISC } \\
\hline Item 1 & & & .706 & & & & & .502 \\
\hline Item 2 & & & .623 & & & & & .612 \\
\hline \multicolumn{9}{|l|}{ ASC } \\
\hline Item 1 & & & & .458 & & & & .791 \\
\hline Item 2 & & & & .810 & & & & .344 \\
\hline \multicolumn{9}{|l|}{ SSC } \\
\hline Item 1 & & & & & .619 & & & .617 \\
\hline Item 2 & & & & & .609 & & & .629 \\
\hline \multicolumn{9}{|l|}{ ESC } \\
\hline Item 1 & & & & & & .765 & & .414 \\
\hline Item 2 & & & & & & .662 & & .562 \\
\hline \multicolumn{9}{|l|}{ CSC } \\
\hline Item 1 & & & & & & & .834 & .305 \\
\hline Item 2 & & & & & & & .768 & .411 \\
\hline \multicolumn{9}{|c|}{ Factor correlations } \\
\hline \multicolumn{9}{|l|}{ GSC } \\
\hline RSC & .124 & & & & & & & \\
\hline ISC & .173 & .456 & & & & & & \\
\hline ASC & .281 & .142 & .172 & & & & & \\
\hline SSC & .391 & .071 & .108 & .500 & & & & \\
\hline ESC & .378 & .197 & .230 & .414 & .747 & & & \\
\hline CSC & .192 & .054 & .134 & .222 & .368 & .386 & & \\
\hline
\end{tabular}

Factor loadings and factor correlations are presented in a standardized form. All parameter estimates are significant at the .05 level. $N=28,824$; GSC: general self-concept; RSC: realistic self-concept; ISC: investigative self-concept; ASC: artistic self-concept; SSC: social self-concept; ESC: enterprising selfconcept; CSC: conventional self-concept.

4.2. GSC as the Predictor of Correlated FSCs. In Model B (Figure 1), GSC is defined by three items and each of the six FSCs is defined by two items, by which GSC can be a pure predictor of FSCs in a path model. In addition, FSCs are set correlated. Model B is examined using SEM, and the results suggest that Model $\mathrm{B}$ fits the overall student sample well $(\mathrm{CFI}=.993$, NNFI $=.989$, and RMSEA $=.022)($ Table 4$)$, a result supporting Hypothesis 4.

Multigroup SEM is performed on Model B across genders, and the results show that females and males are different in the patterns of Model B (CFI $=.842$, NNFI $=.838$, and RMSEA $=.078)$, a result supporting Hypothesis 5. Totalgroup SEM is also performed on Model B for both genders. The results show that Model B fits both females (CFI $=.994$, NNFI $=.991$, and RMSEA $=.019)$ and males $(\mathrm{CFI}=.992$, 
TABLE 3: Factor loadings and goodness-of-fit indexes obtained by HCFA based on the model of GSC as the higher-order factor of FSCs (Model A in Figure 1).

\begin{tabular}{|c|c|c|c|c|c|c|c|c|c|c|c|c|}
\hline \multirow{2}{*}{ Sample } & \multirow[b]{2}{*}{$\mathrm{N}$} & \multicolumn{6}{|c|}{ Factor loadings of GSC defined by } & \multicolumn{5}{|c|}{ Goodness of fit } \\
\hline & & RSC & ISC & ASC & SSC & ESC & CSC & $\chi^{2}$ & $\mathrm{df}$ & CFI & NNFI & RMSEA \\
\hline All & 28824 & .215 & .267 & .524 & .854 & .860 & .439 & 3623.707 & 48 & .958 & .942 & .051 \\
\hline All_mgg & 28824 & .215 & .267 & .524 & .854 & .860 & .439 & 20980.148 & 138 & .730 & .742 & .102 \\
\hline All_F & 15792 & .178 & .227 & .483 & .854 & .837 & .415 & 1482.438 & 48 & .965 & .952 & .044 \\
\hline All_M & 13032 & .324 & .338 & .594 & .856 & .878 & .501 & 1374.563 & 48 & .970 & .959 & .046 \\
\hline Engineering & 5929 & .390 & .414 & .628 & .833 & .890 & .560 & 661.317 & 48 & .970 & .959 & .046 \\
\hline Engineering_mgg & 5929 & .390 & .414 & .628 & .833 & .890 & .560 & 1472.261 & 138 & .934 & .936 & .057 \\
\hline Engineering_F & 846 & .306 & .336 & .488 & .788 & .963 & .507 & 122.730 & 48 & .969 & .957 & .043 \\
\hline Engineering_M & 5083 & .427 & .430 & .658 & .837 & .881 & .576 & 599.826 & 48 & .970 & .959 & .048 \\
\hline Natural sci. & 901 & .277 & .288 & .503 & .845 & .871 & .333 & 101.657 & 48 & .979 & .970 & .035 \\
\hline Natural sci._mgg & 901 & .277 & .288 & .503 & .845 & .870 & .333 & 329.514 & 138 & .908 & .912 & .056 \\
\hline Natural sci._F & 312 & .156 & .218 & .412 & .878 & .754 & .378 & 59.337 & 48 & .982 & .976 & .028 \\
\hline Natural sci._M & 589 & .304 & .307 & .553 & .835 & .901 & .363 & 74.654 & 48 & .985 & .979 & .031 \\
\hline Education & 1026 & .117 & .184 & .514 & .849 & .843 & .335 & 216.938 & 48 & .942 & .920 & .059 \\
\hline Education_mgg & 1026 & .117 & .184 & .514 & .849 & .843 & .335 & 1205.512 & 138 & .696 & .709 & .123 \\
\hline Education_F & 726 & .211 & .149 & .481 & .810 & .874 & .332 & 115.441 & 48 & .961 & .946 & .044 \\
\hline Education_M & 300 & .217 & .381 & .552 & .874 & .815 & .356 & 106.763 & 48 & .944 & .922 & .064 \\
\hline Business & 6629 & .250 & .309 & .500 & .837 & .873 & .422 & 842.658 & 48 & .957 & .941 & .050 \\
\hline Business_mgg & 6629 & .250 & .309 & .500 & .837 & .873 & .422 & 5652.746 & 138 & .745 & .756 & .110 \\
\hline Business_F & 4772 & .204 & .287 & .476 & .853 & .866 & .430 & 478.595 & 48 & .964 & .950 & .043 \\
\hline Business_M & 1857 & .320 & .332 & .574 & .819 & .869 & .500 & 303.767 & 48 & .959 & .944 & .054 \\
\hline
\end{tabular}

HCFA: hierarchical confirmatory factor analysis; GSC: general self-concept; FSCs: field-specific self-concepts, including RSC, ISC, ASC, SSC, ESC, and SSC here; RSC: realistic self-concept; ISC: investigative self-concept; ASC: artistic self-concept; SSC: social self-concept; ESC: enterprising self-concept; CSC: conventional self-concept; F: female; M: male; mgg: multigroup SEM with all parameter estimates being set invariant across genders; mgf: multigroup SEM with all parameter estimates being set invariant across four fields of study (engineering, natural sciences, education, and business). Natural sci.: natural sciences. The bold factor loadings and $\chi^{2}$ are not significant at the .05 level.

NNFI $=.988$, and RMSEA $=.023)$. A closer look at the path coefficients reveals that males have higher effects (path coefficients) of GSC on RSC, ISC, and ASC (.160, .190, and $.293)$ than females $(.084, .161$, and .286), while females have higher effects of GSC on SSC, ESC, and CSC (.409, .384, and $.208)$ than males $(.379, .372$, and .190$)$. The results suggest that GSC is a database of gender roles. Males' GSC predicts FSCs in male-dominated areas (especially, RSC and ISC) more than females, and females' GSC predicts FSCs in femaledominated areas (especially, SSC and CSC) more than males.

Hypothesis 6 predicts that females from different fields of study have different patterns of Model B and males from different fields of study have similar patterns of Model B. The results of multigroup SEM across genders reveal that females from the four fields of study (engineering, natural sciences, education, and business) are different in Model B $(\mathrm{CFI}=.881<.900, \mathrm{NNFI}=.894<.900$, though $\mathrm{RMSEA}=$ $.082<.100)$, while males from the four fields are similar $(\mathrm{CFI}=.953>.900, \mathrm{NNFI}=.958>.900$, and RMSEA $=$ $.046<.100)$, a result supporting Hypothesis 6 . In addition, the results of multigroup SEM across genders for each of the four fields reveal (1) that the pattern of Model B is similar for females and males studying engineering $(\mathrm{CFI}=.964$, NNFI $=$ .963 , and RMSEA $=.041)$ and natural sciences $(\mathrm{CFI}=.939$, $\mathrm{NNFI}=.937$, and RMSEA $=.045$ ), and (2) that the pattern of Model B is different between females and males who study education $(\mathrm{CFI}=.845, \mathrm{NNFI}=.841$, though $\mathrm{RMSEA}=.088)$ and business $(\mathrm{CFI}=.853, \mathrm{NNFI}=.849$, though RMSEA $=$ .083). The results imply that females are more sensitive to study contexts than males.

\section{Discussion}

Two hierarchical models of SC are posited and examined using HCFA and SEM, respectively. The results show that both Model A where GSC acts as the higher-order factor of FSCs and Model B where GSC acts as the predictor of correlated FSCs are valid (Figure 1). In addition, there are differences and interactions between genders and fields of study for the two models.

5.1. GSC Can Act as the Higher-Order Factor of FSCs: Gender Differences and Interaction between Genders and Fields of Study. GSC can be virtually and significantly defined by FSCs as indicated by the results obtained by HCFA for all students (supporting Hypothesis 1) (Table 3 and Model A in Figure 1). In other words, the hierarchical structure of SC can be a bottom-up system in the meaning that GSC is the theme of FSCs, although it may seem to be a top-down system in the statistical representation of CFA (cf. Model A in Figure 1). The result is consistent with research findings 
TABLE 4: Path coefficients and goodness-of-Fit indexes obtained by SEM based on the model of GSC as the predictor of correlated FSCs (Model B in Figure 1).

\begin{tabular}{|c|c|c|c|c|c|c|c|c|c|c|c|}
\hline \multirow{2}{*}{ Sample } & \multicolumn{6}{|c|}{ Path coefficients of GSC on } & \multicolumn{5}{|c|}{ Goodness of fit } \\
\hline & RSC & ISC & ASC & SSC & ESC & CSC & $\chi^{2}$ & $\mathrm{df}$ & CFI & NNFI & RMSEA \\
\hline All & .124 & .173 & .281 & .391 & .378 & .192 & 1044.232 & 69 & .993 & .989 & .022 \\
\hline All_mgg & .124 & .173 & .281 & .391 & .378 & .192 & 17928.499 & 204 & .842 & .838 & .078 \\
\hline All_F & .084 & .161 & .286 & .409 & .384 & .208 & 457.890 & 69 & .994 & .991 & .019 \\
\hline All_M & .160 & .190 & .293 & .379 & .372 & .190 & 565.475 & 69 & .992 & .988 & .023 \\
\hline Engineering & .229 & .217 & .265 & .380 & .351 & .212 & 344.444 & 69 & .991 & .986 & .026 \\
\hline Engineering_mgg & .229 & .217 & .265 & .380 & .351 & .212 & 1227.970 & 204 & .964 & .963 & .041 \\
\hline Engineering_F & .162 & .260 & .224 & .425 & .396 & .239 & 85.757 & 69 & .995 & .992 & .017 \\
\hline Engineering_M & .242 & .211 & .275 & .375 & .345 & .211 & 320.597 & 69 & .990 & .986 & .027 \\
\hline Natural sci. & .117 & .304 & .250 & .320 & .349 & .108 & 102.413 & 69 & .991 & .986 & .023 \\
\hline Natural sci._mgg & .117 & .304 & .250 & .320 & .349 & .108 & 392.127 & 204 & .939 & .937 & .045 \\
\hline Natural sci._F & .170 & .295 & .144 & .361 & .394 & .199 & 73.458 & 69 & .994 & .991 & .014 \\
\hline Natural sci._M & .063 & .310 & .307 & .283 & .321 & .083 & 92.617 & 69 & .991 & .986 & .024 \\
\hline Education & .172 & .240 & .378 & .467 & .405 & .159 & 119.943 & 69 & .990 & .985 & .027 \\
\hline Education_mgg & .172 & .240 & .378 & .467 & .405 & .159 & 1011.146 & 204 & .845 & .841 & .088 \\
\hline Education_F & .142 & .243 & .381 & .514 & .400 & .145 & 109.807 & 69 & .987 & .980 & .029 \\
\hline Education_M & .275 & .328 & .337 & .388 & .417 & .210 & 92.040 & 69 & .986 & .978 & .033 \\
\hline Business & .122 & .177 & .287 & .398 & .411 & .207 & 260.989 & 69 & .993 & .990 & .020 \\
\hline Business_mgg & .122 & .177 & .287 & .398 & .411 & .207 & 4877.513 & 204 & .853 & .849 & .083 \\
\hline Business_F & .086 & .169 & .294 & .396 & .395 & .244 & 198.855 & 69 & .993 & .989 & .020 \\
\hline Business_M & .114 & .152 & .299 & .407 & .429 & .179 & 133.818 & 69 & .993 & .989 & .022 \\
\hline
\end{tabular}

SEM: structural equation modeling; GSC: general self-concept; FSCs: field-specific self-concepts, including RSC, ISC, ASC, SSC, ESC, and SSC here; RSC: realistic self-concept; ISC: investigative self-concept; ASC: artistic self-concept; SSC: social self-concept; ESC: enterprising self-concept; CSC: conventional selfconcept; F: female; M: male; mgg: multigroup SEM with all parameter estimates being set invariant across genders; mgf: multigroup SEM with all parameter estimates being set invariant across four fields of study (engineering, natural sciences, education, and business). Natural sci.: natural sciences. The bold path coefficients and $\chi^{2}$ are not significant at the .05 level.

obtained by the same statistical methods in related studies $[16,17]$. Shavelson et al.'s [12] theoretical model is a large and detailed system and is hard to be completely examined and addressed at the same time based on empirical data (Marsh et al. [16]). However, the essence of Shavelson et al.'s model is normally supported. There is a highest-order GSC and lowerorder FSCs, which highlight the generality of GSC and the specificity of FSCs. The model that GSC acts as the higherorder factor of FSCs indicates that FSCs are reflections of selfknowledge in response to world knowledge and GSC is an abstraction of these reflections.

Females and males appear to be different in the patterns of abstraction from FSCs to GSC (supporting Hypothesis 2). In addition, there appears to be a trend that males are stronger in the abstraction (factor loadings) than females. In other words, males view FSCs as more similar than females, or females can distinguish different FSCs better than males. A similar result is found by Marsh [42] that gender differences are significant, though small, in domain-specific attitudes.

Gender differences in the patterns of abstraction from FSCs to GSC are also subject to fields of study (supporting Hypothesis 3). Males from both male-dominated fields (engineering and natural sciences) and female-dominated fields (education and business) are similar in the pattern of the abstraction. However, females studying male-dominated fields are different from females studying female-dominated fields in their patterns. Further, females and males studying male-dominated fields are similar, while females and males studying female-dominated fields are different. If we use males as a control group because males are similar no matter what their fields of study are, then we can infer that females studying female-dominated fields are unique and females studying male-dominated domains are likely to be reflective of the pattern of males. A reason for this result is likely to be females' sensitivity to social expectations and pressure, by which females become similar to males after a three-year study in male-dominated fields, that is, the socialization assumption. It is also likely that females go for male-dominated fields because of their similarity with males in the patterns of abstraction from FSCs to GSC, that is, the congruence assumption [34]. Further research can address this issue more.

\subsection{GSC Can Act as the Predictor of Correlated FSCs: Gender} Differences and Interaction between Genders and Fields of Study. GSC can also act as the predictor of correlated FSCs, as indicated by the results obtained by SEM for all students (supporting Hypothesis 4) (Table 4 and Model B in Figure 1). In other words, the hierarchical structure of SC can be a topdown system: GSC can substantially exert influence on FSCs. This forms a new way to explain and validate Shavelson et al.'s [12] hierarchical structure of SC. Compared with the results in 
relation to Hypothesis 1 , we can find that Model B is slightly better than Model A in terms of the values of fit indexes as Model B's CFI and NNFI are larger, and RMSEA is smaller $(\mathrm{CFI}=.993, \mathrm{NNFI}=.989$, and RMSEA $=.022$ in Table 4$)$ than Model A's $(\mathrm{CFI}=.958$, NNFI $=.942$, and RMSEA $=.051$ in Table 3 ). The results suggest that the hierarchical structure of SC as a path model should have an indispensable position in the interpretation of Shavelson et al's theory.

Gender differences play a role in the way that GSC leads FSCs, as indicated by multigroup SEM across genders (supporting Hypothesis 4). There is also a slight trend that females' GSC exerts influence on SCs in social, enterprising, and conventional areas more than males'. On the other hand, males' GSC exerts influence on SCs in realistic, investigative, and artist areas more than females. The results imply that GSC as a leader may function in a way of reflecting longterm social expectations of gender roles. Marsh's [42] research also indicates that gender differences in academic attitudes are consistent with gender stereotypes, with males having more positive attitudes towards math and females having more positive attitudes towards verbal skills. A model where GSC acts as the predictor of correlated FSCs can reflect the phenomenon of socialization of gender roles.

Like Model A (Hypothesis 3), gender differences in Model $\mathrm{B}$ are subject to fields of study (Hypothesis 6). Females from female-dominated fields (education and business) are different from those in male-dominated fields (engineering and natural sciences), while males from the four fields are similar in Model B. The results are attributed to females' higher degree of sensitivity to short-term social messages or expectations than males.

A note to make is that business is viewed as a female domain and more females study business in Taiwan, but business is viewed as a male domain in the USA. The reason for this difference may be that business is viewed as being related to mathematics in the USA [43]. In Taiwan, highschool students aim to study business in university choose the social sciences course package, including social-science mathematics courses and take social-science mathematics tests in their college entrance examination. Natural-science mathematics courses and tests are taken by students who aim to study mathematics, natural/medical sciences, and engineering.

5.3. Limitations of the Present Study and Implications for Future Research. The use of very few (one or two) items for one psychological construct appears to violate psychometric tradition but is necessary for daily practice [44]. Similar rationales can be found for large-scale surveys, in which many kinds of constructs of data have to be collected to address multiple research issues. Reasonable results are obtained for the present models examined in terms of theory, which suggests an acceptable use of very few items for one construct when the sample size is large. This, however, should be validated by future research. The notion that the hierarchical structure of SC is likely to be stable, developmental, and evaluative can be explored with longitudinal data [22, 45]. Students in higher education and those in primary and secondary education are likely to have different components and patterns in the hierarchical structure of SC. For instance, female students in secondary education generally have a lower academic self-concept than their male counterparts. The generalization of the present findings regarding the differential validity of the three hierarchical models of SC across genders and fields of study in Taiwan needs to be examined by research for students from other cultures.

\section{References}

[1] B. N. Githua and J. G. Mwangi, "Students' mathematics selfconcept and motivation to learn mathematics: relationship and gender differences among Kenya's secondary-school students in Nairobi and Rift Valley provinces," International Journal of Educational Development, vol. 23, no. 5, pp. 487-499, 2003.

[2] H. W. Marsh, R. G. Craven, and D. M. McInerney, Eds., International Advances in Self Research, Information Age, Greenwich, Conn, USA, 2003.

[3] B. M. Byrne, "Measuring adolescent self-concept: factorial validity and equivalency of the SDQ III across gender," Multivariate Behavioral Research, vol. 23, no. 3, pp. 361-375, 1988.

[4] H. W. Marsh and R. O’Neill, “Self Description Questionnaire III: the construct validity of multidimensional self-concept ratings by late adolescents," Journal of Educational Measurement, vol. 21, no. 2, pp. 153-174, 1984.

[5] K. Cokley and N. Patel, "A psychometric investigation of the academic self-concept of Asian American college students," Educational and Psychological Measurement, vol. 67, no. 1, pp. 88-99, 2007.

[6] K. El-Hassan, "Structure and correlates of self-concept in Lebanon," International Journal of Testing, vol. 4, no. 1, pp. 1-17, 2004.

[7] S. Nishikawa, T. Norlander, P. Fransson, and E. Sundbom, "A cross-cultural validation of adolescent self-concept in two cultures: Japan and Sweden," Social Behavior and Personality, vol. 35, no. 2, pp. 269-286, 2007.

[8] S. F. Tam and D. Watkins, "Development and validation of a selfconcept questionnaire for Hong Kong Chinese adults who have physical disability," Social Behavior and Personality, vol. 25, no. 1, pp. 1-12, 1997.

[9] A. S. F. Tam and D. Watkins, "Towards a hierarchical model of self-concept for Hong Kong Chinese adults with physical disabilities," International Journal of Psychology, vol. 30, no. 1, pp. 1-17, 1995.

[10] P. Yin and X. Fan, "Assessing the factor structure invariance of self-concept measurement across ethnic and gender groups: findings from a national sample," Educational and Psychological Measurement, vol. 63, no. 2, pp. 296-318, 2003.

[11] C. H. K. Cheng and D. Watkins, "Age and gender invariance of self-concept factor structure: an investigation of a newly developed Chinese self-concept instrument," International Journal of Psychology, vol. 35, no. 5, pp. 186-193, 2000.

[12] R. J. Shavelson, J. J. Hubner, and G. C. Stanton, "Self-concept: validation of construct interpretations," Review of Educational Research, vol. 46, no. 3, pp. 407-441, 1976.

[13] H. W. Marsh, "The hierarchical structure of self-concept and the application of hierarchical confirmatory factor analysis," Journal of Educational Measurement, vol. 24, no. 1, pp. 17-39, 1987.

[14] H. W. Marsh, "The multidimensional structure of academic selfconcept: invariance over gender and age," American Educational Research Journal, vol. 30, no. 4, pp. 841-860, 1993. 
[15] H. W. Marsh and A. S. Yeung, "Coursework selection: relations to academic self-concept and achievement," American Educational Research Journal, vol. 34, no. 4, pp. 691-720, 1997.

[16] H. W. Marsh, B. M. Byrne, and R. J. Shavelson, "A multifaceted academic self-concept: Its hierarchical structure and its relation to academic achievement," Journal of Educational Psychology, vol. 80, no. 3, pp. 366-380, 1988.

[17] H. W. Marsh, U. Trautwein, O. Lüdtke, O. Köller, and J. Baumert, "Integration of multidimensional self-concept and core personality constructs: construct validation and relations to well-being and achievement," Journal of Personality, vol. 74, no. 2, pp. 403-456, 2006.

[18] H. W. Marsh and R. J. Shavelson, "Self-concept: its multifaceted, hierarchical structure," Educational Psychologist, vol. 20, no. 3, pp. 107-125, 1985.

[19] H. W. Marsh, "A multidimensional, hierarchical model of selfconcept: theoretical and empirical justification," Educational Psychology Review, vol. 2, no. 2, pp. 77-172, 1990.

[20] H. W. Marsh, "The structure of academic self concept: the Marsh/Shavelson model," Journal of Educational Psychology, vol. 82, no. 4, pp. 623-636, 1990.

[21] H. W. Marsh, R. Craven, and R. Debus, "Separation of competency and affect components of multiple dimensions of academic self-concept: a developmental perspective," MerrillPalmer Quarterly, vol. 45, no. 4, pp. 567-601, 1999.

[22] H. W. Marsh and A. S. Yeung, "Longitudinal structural equation models of academic self-concept and achievement: gender differences in the development of math and English constructs," American Educational Research Journal, vol. 35, no. 4, pp. 705738,1998

[23] H. W. Marsh, "The factorial invariance of responses by males and females to a multidimensional self-concept instrument: substantive and methodological issues," Multivariate Behavioral Research, vol. 22, no. 4, pp. 457-480, 1987.

[24] M. del Prado Silván-Ferrero and A. B. López, "Benevolent sexism toward men and women: justification of the traditional system and conventional gender roles in Spain," Sex Roles, vol. 57, no. 7-8, pp. 607-614, 2007.

[25] S. Brownlow, T. J. Smith, and B. R. Ellis, "How interest in science negatively influences perceptions of women," Journal of Science Education and Technology, vol. 11, no. 2, pp. 135-144, 2002.

[26] M. S. Chiu, "Effects of a women-in-sciences/men-in-humanities intervention on Taiwanese adolescents'attitudes towards learning science," Asia-Pacific Education Researcher, vol. 20, pp. 322-335, 2011.

[27] N. Zhang, "Gender role egalitarian attitudes among Chinese college students," Sex Roles, vol. 55, no. 7-8, pp. 545-553, 2006.

[28] C. Morgan, J. D. Isaac, and C. Sansone, "The role of interest in understanding the career choices of female and male college students," Sex Roles, vol. 44, no. 5-6, pp. 295-320, 2001.

[29] J. F. Hair Jr., W. C. Black, B. J. Babin, R. E. Anderson, and R. L. Tatham, Multivariate Data Analysis, Prentice-Hall, Upper Saddle River, NJ, USA, 6th edition, 2006.

[30] H. W. Marsh and K. T. Hau, "Explaining paradoxical relations between academic self-concepts and achievements: crosscultural generalizability of the internal/external frame of reference predictions across 26 countries," Journal of Educational Psychology, vol. 96, no. 1, pp. 56-67, 2004.

[31] M. S. Chiu, "Achievements and self-concepts in a comparison of math and science: exploring the internal/external frame of reference model across 28 countries," Educational Research and Evaluation, vol. 14, no. 3, pp. 235-254, 2008.
[32] National Taiwan Normal University, The Integrated Higher Education Database System, 2008, https://www.cher.ntnu.edu.tw/.

[33] A. García-Aracil, D. Gabaldón, J. G. Mora, and L. E. Vila, “The relationship between life goals and fields of study among young European graduates," Higher Education, vol. 53, no. 6, pp. 843865, 2007.

[34] K. A. Feldman, J. C. Smart, and C. A. Ethington, "Using Holland's theory to study patterns of college student success: the impact of major fields on students," in Higher Education: Handbook of Theory and Research, J. C. Smart, Ed., pp. 329-380, Springer, Dordrecht, The Netherlands, 2008.

[35] C. P. Deng, P. I. Armstrong, and J. Rounds, "The fit of Holland's RIASEC model to US occupations," Journal of Vocational Behavior, vol. 71, no. 1, pp. 1-22, 2007.

[36] K. G. Joreskog and D. Sorbom, LISREL 8. 80 [Computer Software], Scientific Software International, Lincolnwood, Ill, USA, 2007.

[37] M. Du Toit and S. H. C. Du Toit, Interactive LISREL: User's Guide, Scientific Software International, Lincolnwood, Ill, USA, 2001.

[38] K. G. Jöreskog and D. Sörbom, LISREL 8: User's Reference Guide, Scientific Software International, Lincolnwood, Ill, USA, 2001.

[39] K. A. Bollen and J. S. Long, Testing Structural Equation Models, Sage, Newbury Park, Calif, USA, 1993.

[40] M. W. Browne and R. Cudeck, "Alternative ways of assessing model fit," in Testing Structural Equation Models, K. A. Bollen and J. S. Long, Eds., pp. 136-162, Sage, Newbury Park, Calif, USA, 1993.

[41] R. E. Schumacker and R. G. Lomax, A Beginner's Guide to Structural Equation Modeling, Erlbaum, Mahwah, NJ, USA, 1996.

[42] H. W. Marsh, "Sex differences in the development of verbal and mathematics constructs: the high school and beyond study," American Educational Research Journal, vol. 26, pp. 191-225, 1989.

[43] H. M. Lips, "The gender gap in possible selves: divergence of academic self-views among high school and university students," Sex Roles, vol. 50, no. 5-6, pp. 357-371, 2004.

[44] S. D. Gosling, P. J. Rentfrow, and W. B. Swann Jr., "A very brief measure of the big-five personality domains," Journal of Research in Personality, vol. 37, no. 6, pp. 504-528, 2003.

[45] A. S. Yeung and F. L. Lee, "Self-concept of high school students in China: confirmatory factor analysis of longitudinal data," Educational and Psychological Measurement, vol. 59, no. 3, pp. 431-450, 1999. 

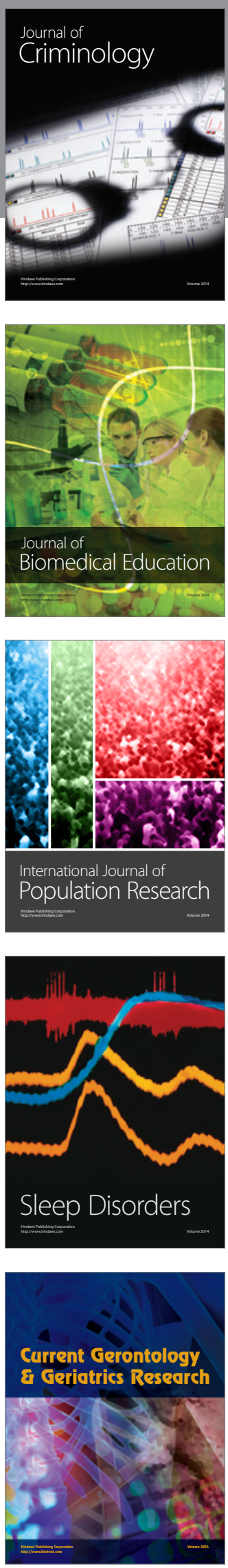
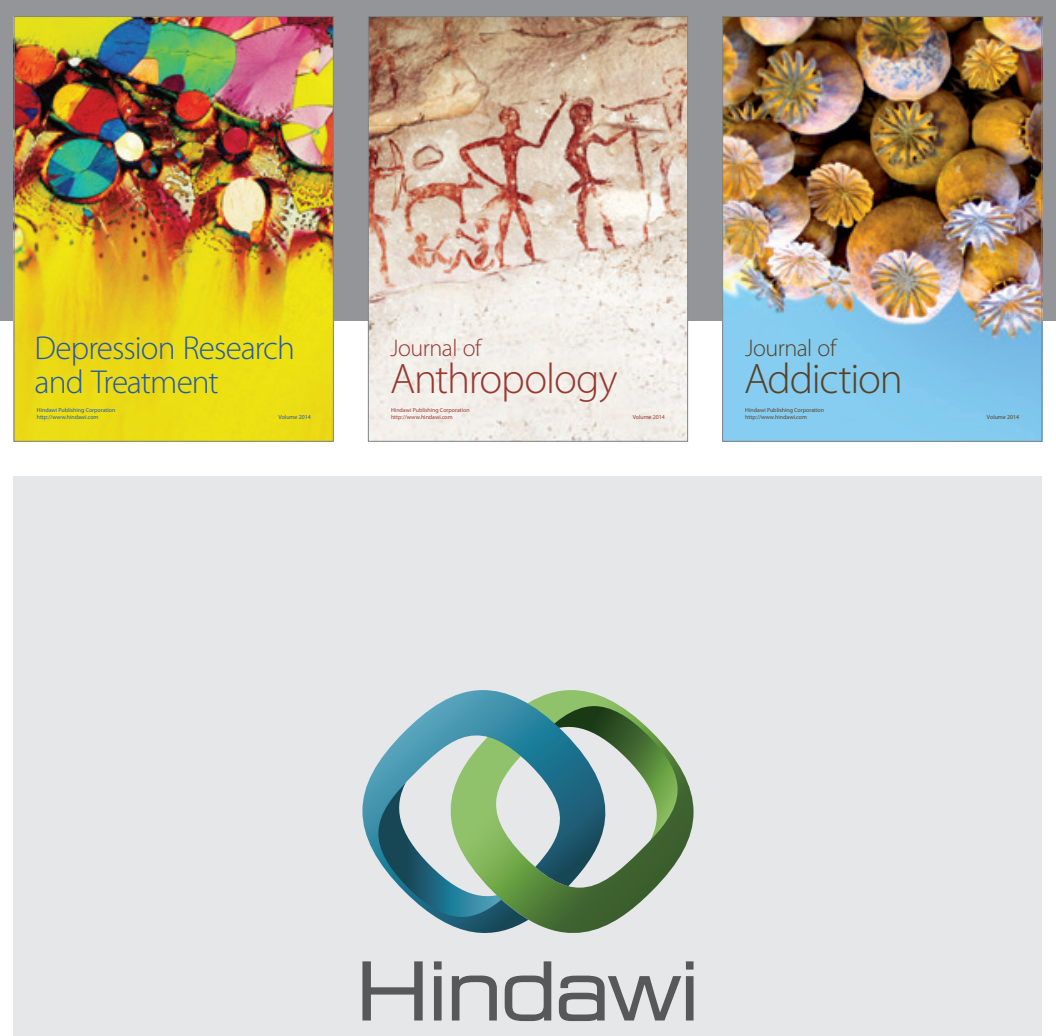

Submit your manuscripts at

http://www.hindawi.com

Child Development Research
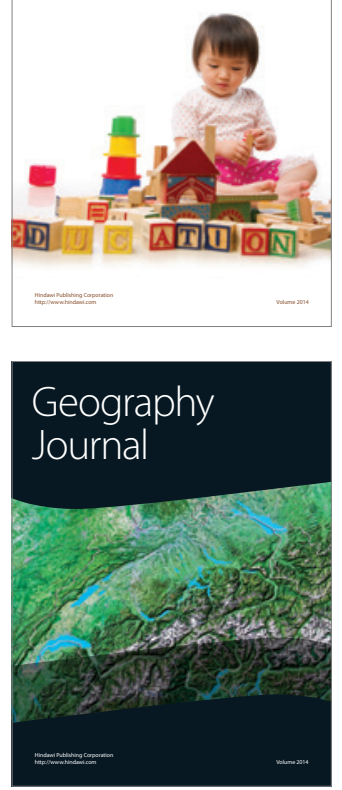

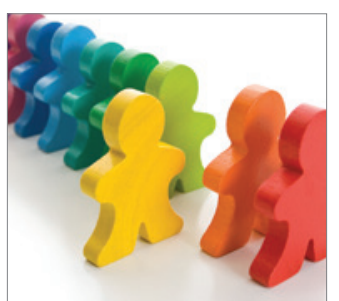

Autism

Research and Treatment
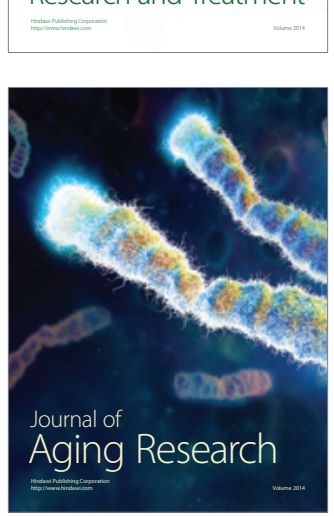
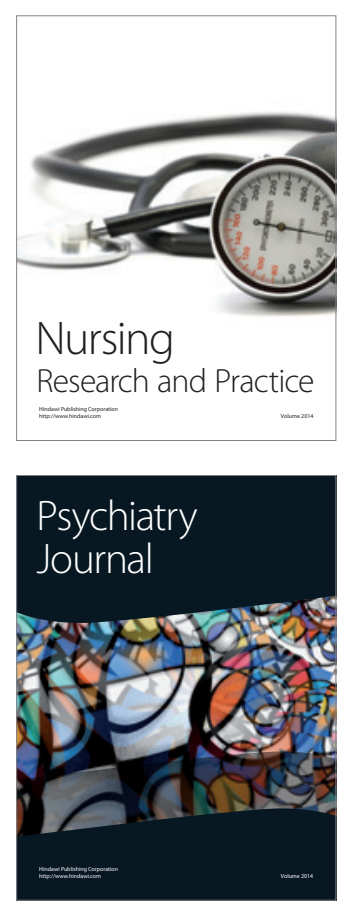
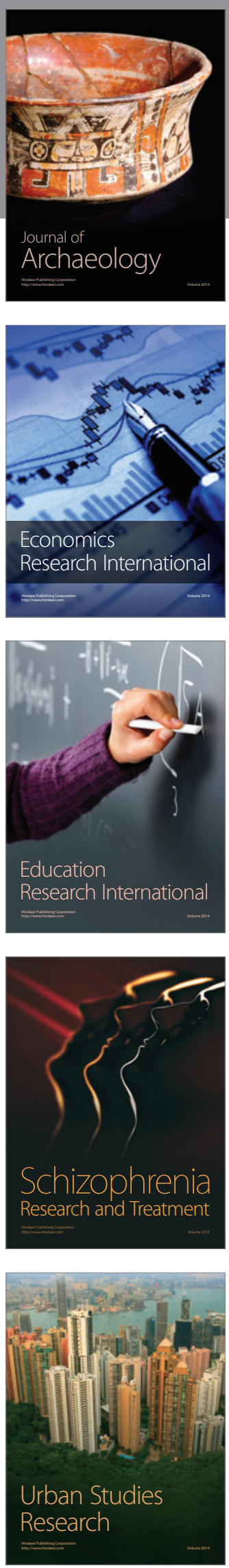